\title{
HIGH-RESOLUTION STRAIN SENSING ON STEEL BY SILICON-ON-INSULATOR FLEXURAL RESONATORS FABRICATED WITH CHIP-LEVEL VACUUM PACKAGING
}

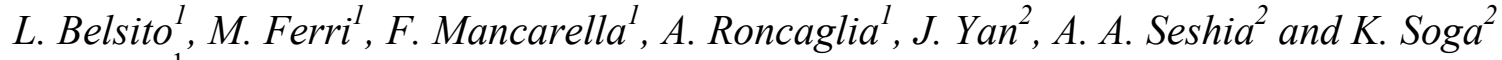 \\ ${ }^{1}$ Institute of Microelectronics and Microsystems, CNR, Bologna, Italy \\ ${ }^{2}$ Department of Engineering, University of Cambridge, Cambridge, United Kingdom
}

\begin{abstract}
This paper reports on the fabrication and characterization of high-resolution strain sensors for steel based on Silicon On Insulator flexural resonators manufactured with chip-level LPCVD vacuum packaging. The sensors present high sensitivity $(120 \mathrm{~Hz} / \mu \varepsilon)$, very high resolution $\left(\begin{array}{ll}4 & \mathrm{n} \varepsilon\end{array}\right)$, low drift, and near-perfect reversibility in bending tests performed in both tensile and compressive strain regimes.
\end{abstract}

\section{KEYWORDS}

Strain sensors, flexural resonators, SOI technology, MEMS, chip-level vacuum packaging.

\section{INTRODUCTION}

The use of Micro-Electro-Mechanical flexural resonators as ultra high resolution strain sensors for structural materials has been recently proposed and demonstrated both with devices operating at atmospheric pressure [1] and with devices operating in vacuum [2].

Vacuum operation promises better performance in this sensing context, due to the higher resonator $\mathrm{Q}$ factor that can be achieved and the consequent benefits that this property brings along in terms of phase noise and power dissipation for the closed-loop operation of such devices. However, a durable vacuum packaging on strain sensors bonded on steel is not easily achievable by hybrid packaging techniques, as described in previous research [2].

In this paper, the problem of vacuum packaging of the resonators is addressed by packaging devices fabricated on Silicon On Insulator (SOI) substrates with Low-Pressure Chemical Vapour Deposition (LPCVD) based vacuum packaging. This technique, already demonstrated for MEMS oscillators [3], is here proposed for the first time in the field of resonant strain sensors.

\section{FABRICATION}

The MEMS strains sensors were fabricated starting from SOI substrates with $25 \mu \mathrm{m}$ thick, n-type doped device layer and $2 \mu \mathrm{m}$ buried oxide thickness.

After front-side micromachining of the devices, a classical LPCVD sealing technology based on polysilicon deposition is used for chip-level vacuum packaging of the devices [3]. The final result of the fabrication is shown in cross-section in Fig. 1 (top of the figure). As may be observed, the lateral flexural resonator used for strain sensing is encapsulated within a thick polysilicon shield which maintains a stable vacuum level inside the cavity, enabling high-performance operation of the resonator itself.

Several resonator geometries have been fabricated with the described process, based on Double-EndedTuning-Fork (DETF) topologies integrated with parallelplate and comb electrodes and air gaps between 1.5 and 2 $\mu \mathrm{m}$ in width. In Fig. 1 (bottom part of the figure), a comb electrode DETF resonator geometry is reported by way of example.
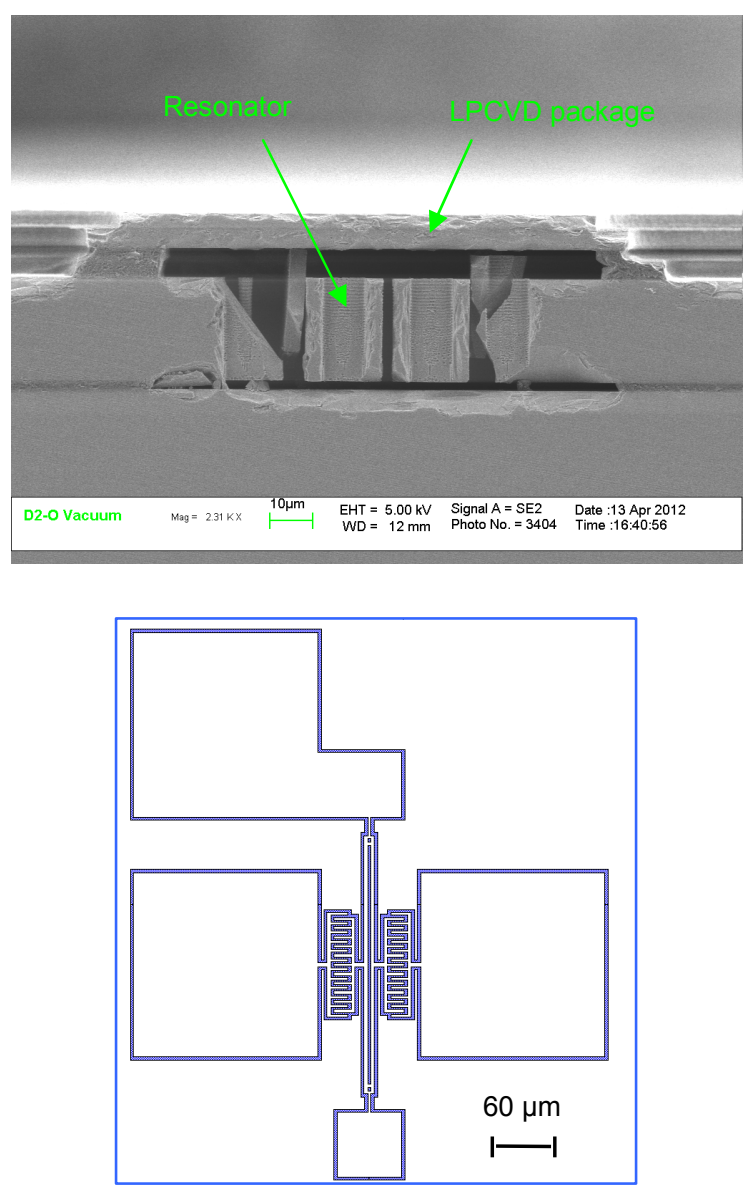

Figure 1: Cross-section of parallel-plate DETF flexural resonator fabricated with chip-level vacuum packaging by LPCVD sealing (top) and sample layout of comb DETF resonator (bottom).

In Fig. 2, the fabrication process of the resonators with LPCVD vacuum sealing is schematically represented.

After conventional SOI micromachining by Deep Reactive Ion Etching (DRIE) of the $25 \mu \mathrm{m}$ thick device layer (steps 1-2), the patterned geometry is planarized by LPCVD $\mathrm{SiO}_{2}$ deposition (step 3) and covered by a $7 \mu \mathrm{m}$ thick polysilicon layer (step 4). On the polysilicon shield, small apertures are then created by RIE etching (step 5), 
through which the SOI structure is released from the substrate through Hydrofluoric Acid (HF) vapour etching (step 6). After release, the polysilicon shield is sealed by another LPCVD polysilicon deposition $(3 \mu \mathrm{m})$, in order to eventually vacuum seal the cavity (step 7). Finally, the polysilicon shield and the underlying $\mathrm{SiO}_{2}$ layer are etched on the bonding pad locations for the resonators to allow device wiring (step 8).
1. SOI wafer
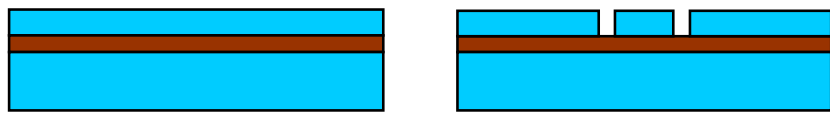

5. Polysilicon etching

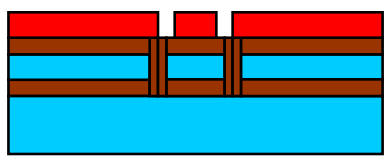

6. $\mathrm{SiO}_{2} \mathrm{HF}$ vapour etching

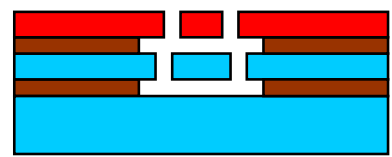

3. $\mathrm{SiO}_{2}$ deposition

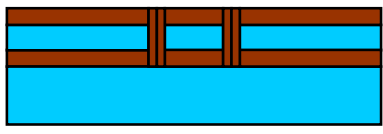

7. Polysilicon deposition

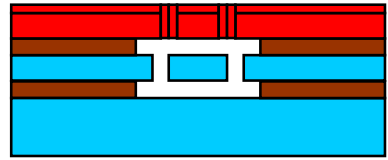

\section{Polysilicon deposition}

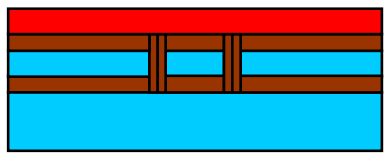

8. Poly/SiO 2 etching

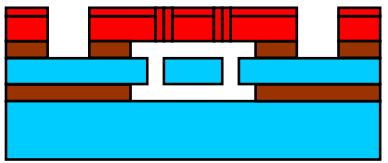

Figure 2: Fabrication process of the SOI flexural resonators with LPCVD vacuum packaging.

\section{TESTING}

\section{Open-loop testing}

The manufactured devices were initially tested in open loop using a setup composed of a network analyzer (Anritsu MS2036C) and a transresistance amplifier connected in a two-port configuration of the mobile and fixed electrodes of the devices under measurement. In order to investigate the yield of the fabrication process, a number of devices (175) on a processed wafer were tested in open-loop with the described setup.

The measurement results evidenced a low process yield (only about $28 \%$ over the total number of tested devices showed a clear resonance peak under stable vacuum). The reason for this low yield was not entirely clear and is currently under further investigation.

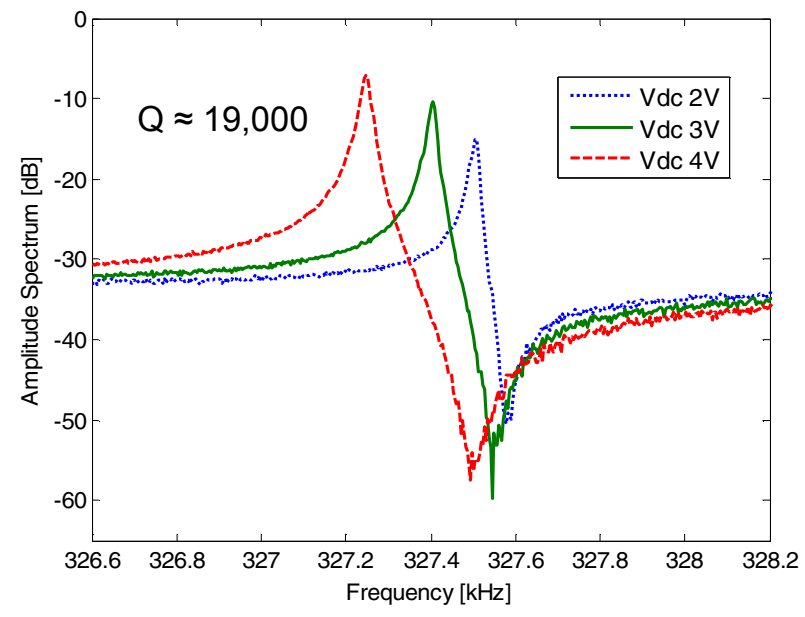

Figure 3: Open-loop measurements on LPCVD-sealed parallel-plate DETF resonator with $1.5 \mu \mathrm{m}$ air gap.
Despite the poor results obtained in terms of yield, the working devices on the sample showed very good open-loop characteristics, with low feedthrough and resonance peaks up to $25 \mathrm{~dB}$ in height (see for instance the open-loop measurement performed on a parallelplate device reported in Fig. 3).

The open-loop measurements were analyzed further to evaluate the $\mathrm{Q}$ factor of the devices (found to be in the range 10,000-20,000) and, by comparison with similar measurements performed on unpackaged resonators from the same processed sample, the vacuum level reached within the LPCVD sealing is estimated to be around 120 mTorr.

\section{Functional testing}

The devices were tested in closed loop using a previously-designed readout circuit composed of a transresistance amplifier-based oscillator and a microcontroller-based digital frequency counter (previously presented in [2]), and a bending test setup consisting of a $1.5 \mathrm{~mm}$ thick steel cantilever onto which the resonator chip was bonded by epoxy glue (Torr Seal from Varian), see Fig. 4.

Load tests were performed by controlled inflection of the steel cantilever operated by a micrometric screw. From the inflection, the strain applied on the sensor under test was derived from the analytical formulation for beam bending [4], and the sensitivity calculated by the ratio of the measured frequency shift on the closed loop operated devices and the applied strain level.

In Fig. 5, a calibration curve derived from load test results obtained on a comb type DETF resonator operating around $268 \mathrm{kHz}$ is reported. As may be seen, a high sensitivity around $120 \mathrm{~Hz} / \mu \varepsilon$ is obtained, both in tensile and compressive strain regime. 


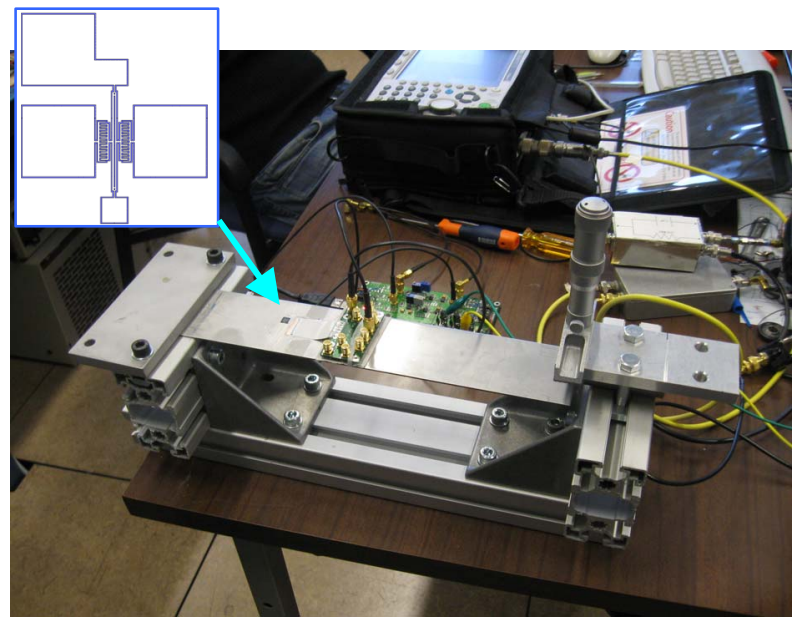

Figure 4: Bending test setup for the strain sensors with tested resonator geometry (top-left of the figure).

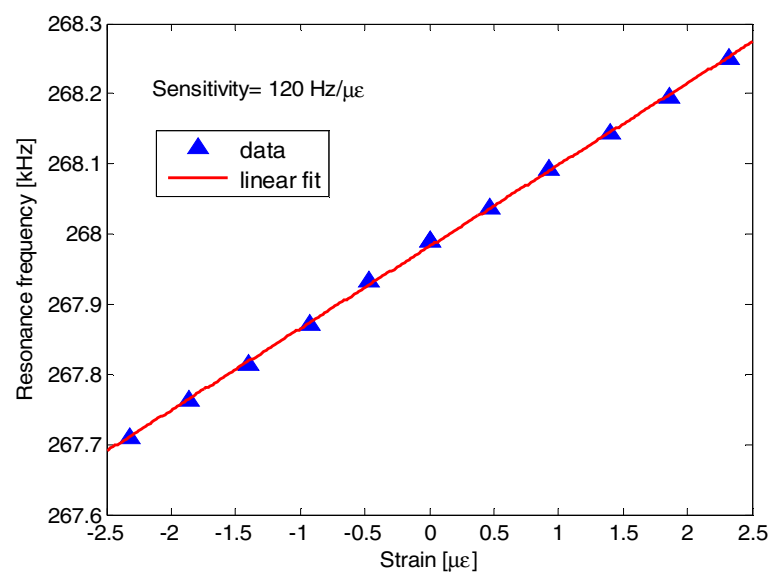

Figure 5: Calibration curve obtained by the load test on the comb resonator in tensile and compressive strain.

Moreover, as can be seen in Fig. 6 in which a representative load test at increasing strain levels is reported, the sensor response shows almost perfect reversibility and low drift.

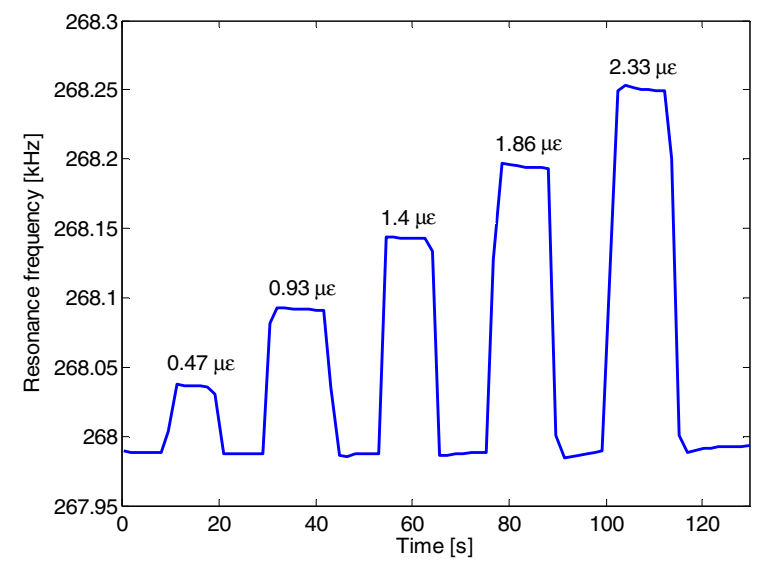

Figure 6: Load test on LPCVD-sealed comb DETF resonator with $2 \mu \mathrm{m}$ air gap operating in closed loop.

In order to estimate the resolution of the sensor in terms of applied strain, the noise on the output of the closed-loop circuit was evaluated by measuring the short-term $(1 \mathrm{~min})$ frequency jitter at room temperature (Fig. 7).

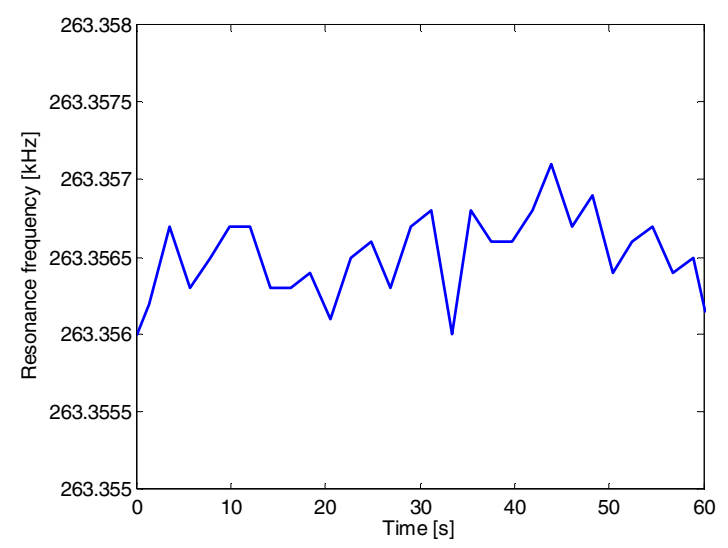

Figure 7: Evaluation of short-term (60 sec) frequency instability on the output of the sensor readout circuitry.

As may be seen from the plot, the short-term frequency instability turns out to be around $0.5 \mathrm{~Hz}$, yielding a resolution limit around $4 \mathrm{n} \varepsilon$ for the sensor (considering the sensitivity of $120 \mathrm{~Hz} / \mu \varepsilon$ derived from the calibration curve shown in Fig. 5).

A further test was performed by acquiring the sensing signal on two resonators located on the same steel-bonded chip (Fig. 8), in order to evaluate the performance of possible temperature compensation schemes to compensate the thermal drift of the strain sensors (owing to both well-established inherent thermal drift mechanisms and due to the thermal expansion coefficient mismatch between steel and silicon).

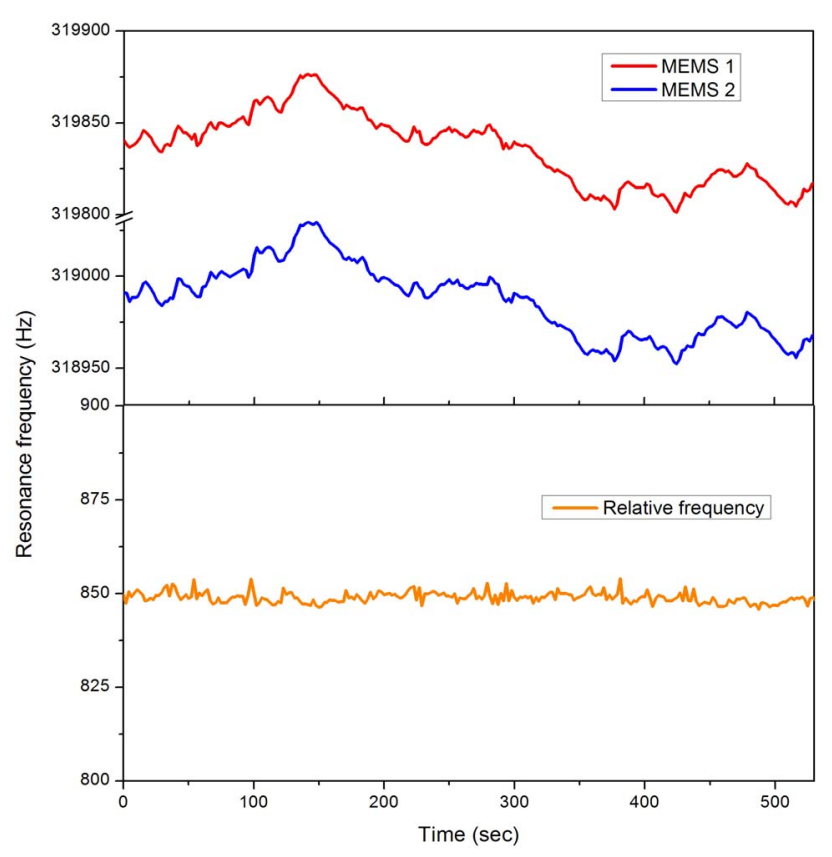

Figure 8: Temperature drift compensation scheme based on differential sensing with two identical resonators on the same chip. 
As may be observed, a good suppression of the thermal drift over the observed time interval is possible by using two resonators of the same geometry. This is a promising result in view of possible implementation of compensation schemes based on reference resonators on unstrained parts of the mechanical structure under measurement.

\section{REFERENCES}

[1] A. P. Pisano, "MEMS and Nanotechnology for the Handheld, Portable Electronic and the Automotive Markets", in Digest Tech. Papers Transducers '07 Conference, Lyon, June 10-14, 2007, pp. 1-4.

[2] M. Ferri, F. Mancarella, L. Belsito, A. Roncaglia, J. Yan, A. A. Seshia, K. Soga, J. Zalesky, "Strain sensing on steel surfaces using vacuum packaged MEMS resonators", Procedia Engineering 5 (2010), pp. 1426-1429.

[3] B. Kim, R. N. Candler, M. A. Hopcroft, M. Agarwal, W.-T. Park, T. W. Kenny, "Frequency stability of wafer-scale film encapsulated siliconbased MEMS resonators", Sensors and Actuators A 136 (2007), pp. 125-131.

[4] J. M. Gere, S. P. Timoshenko, Mechanics of Materials, Chap. 10, PWS Publishing Company, 1997.

\section{CONTACT}

*A. Roncaglia, tel: +39-051-6399122; roncaglia@bo.imm.cnr.it 